\title{
El análisis multidimensional de datos: una aplicación al estudio de la relación entre colaboración infantil entre pares y contexto socioeconómico
}

\author{
Juan Manuel Curcio \\ Mariano Castellaro \\ Nadia Soledad Peralta \\ Instituto Rosario de Investigaciones en Ciencias de la Educación \\ Facultad de Psicología Universidad Nacional de Rosario \\ Rosario, Argentina.
}

\author{
Recibido: 10 de febrero del 2018 / Aprobado: 28 de febrero del 2018 \\ doi: https://doi.org/10.26439/persona2018.n021.1994
}

\begin{abstract}
El trabajo propone e ilustra la utilización de la versión francesa del análisis de datos multidimensionales (AMD) como un enfoque alternativo para el análisis de datos cuantitativos en ciencias sociales, particularmente en Psicología. Para tal fin, se recuperó una investigación previa que estudió la interacción colaborativa infantil en una tarea de construcción con bloques, en dos contextos socioeconómicos (CSE): favorecido (CSF) y desfavorecido (CSD). Se analizó la modalidad social de ejecución de la tarea y los tipos de mensaje, y los resultados opusieron el CSF (asociado a modalidades de cooperación y cooperación explícita) al CSD (vinculado con la cooperación implícita). Estos resultados coinciden con los estudios previos, ya que los sujetos de ambos CSE registraron niveles similares de coordinación social, aunque la modalidad específica de interacción es diferente. Se destaca el valor de este tipo de herramientas analiticas en el campo de las ciencias sociales por la complejidad que implican sus objetos de estudio y la consiguiente posibilidad de lograr aproximaciones exploratorias y más integrales.
\end{abstract}

análisis de datos multidimensionales / análisis cuantitativo / colaboración entre pares / infancia

Correo electrónico: castellaro@irice-conicet.gov.ar 


\section{Multidimensional Data Analysis: An Application to the Study of the Relationship Between Children's Peer Collaboration and Socioeconomic Context}

This paper proposes and illustrates the use of the French version of the multidimensional data analysis (MDA) as an alternative approach for analyzing quantitative data in social sciences, particularly in psychology. To that end, we revisited a previous research which analyzed children's peer collaboration in a task involving building a house with construction blocks in two socioeconomic contexts (SEC): advantaged socioeconomic context (ASC) and disadvantaged socioeconomic context (DSC). The social modality of task execution and the types of message were analyzed. The results from the ASC (associated with explicit cooperation and collaboration) were contrary to those of the DSC (linked to implicit cooperation). These results are consistent with those from previous studies, for both SECs register similar levels of social coordination, but the specific interaction modality is different. This kind of analytical tools is particularly important in social sciences due to the complexity of the objects of study and the resulting possibility to achieve more comprehensive exploratory approaches.

multidimensional data / quantitative analysis / peer collaboration / childhood 


\section{INTRODUCCIÓN}

\section{Contexto, contexto socioeconómico y colaboración infantil entre pares}

La relación entre interacción social, contexto y desarrollo infantil es un punto teórico central en Psicología, especialmente para aquellas perspectivas fuertemente comprometidas con una concepción social de la ontogénesis. El socioconstructivismo es un claro exponente de ello (por ejemplo, Psaltis, Duveen y Perret Clermont, 2009; Rogoff, 1990), al sostener dos ideas principales (entre otras no menos importantes). En primer lugar, la interacción social o intersubjetividad es la base de los cambios cualitativos del desarrollo, especialmente en sus aspectos cognitivos. En este sentido, la mayoría de las investigaciones se centraron en demostrar la superioridad de los procesos sociocognitivos en comparación con los procesos individuales y el efecto causal de los primeros sobre el desarrollo cognitivo individual. En segundo término, estas formas de interacción social están involucradas en sistemas de actividad específicos, constituidos no solo por los otros significativos y las dinámicas interactivas establecidas con ellos, sino también por las condiciones materiales y concretas sobre las cuales tienen lugar dichos intercambios (por ejemplo, el formato de la tarea).

Esta perspectiva implica un cuestionamiento de los modelos madurativos que enfatizan el carácter endógeno del desarrollo, entendido como una trayectoria uniforme a través de etapas sucesivas, diferenciadas principalmente por edades cronológicas. Una perspectiva social entiende que el desarrollo puede asumir direcciones variables dependiendo del contexto sociocultural específico, ya que este se convierte en un aspecto estructurante del proceso ontogenético. Por lo tanto, el estudio del desarrollo social debe considerar las condiciones contextuales bajo las cuales el individuo se desenvuelve habitualmente.

El concepto de contexto puede asociarse con la idea de variación cultural. Siguiendo a Mejía-Arauz (1999), la precedencia cultural se refiere a una forma de vida compartida entre sujetos, también asociada a formas específicas de interacción social, intersubjetividad y formatos institucionales. Cualquier contexto particular puede definirse por diferentes características sociales comunes, tales como nacionalidad, región de residencia, nivel educativo, creencias y costumbres, entre otras.

Barbara Rogoff y sus continuadores son referentes en el estudio de la relación entre las variaciones culturales y el proceso colaborativo intersubjetivo niño-adulto y niño-niño. Entre otros, Correa-Chávez, Rogoff y Mejía-Arauz (2005) y Mejía-Arauz, Rogoff, Dexter y Najafi (2007) compararon los patrones de organización social infantil espontáneos en familias que eran originalmente indígenas mexicanos inmigrantes y niños estadounidenses descendientes de inmigrantes europeos, en tríadas de seis a diez años. También, Correa-Chávez y Rogoff (2009) estudiaron las diferencias 
concepto de contexto socioeconómico (CSE) desde una perspectiva sociocultural similar. Esto significa que el interés no está tanto en las condiciones reales y objetivas que definen el CSE, sino más bien en los aspectos culturales y relacionales asociados a esos indicadores externos. Siguiendo a Rosemberg, Menti, Stein, Alam y Migdalek (2016), la noción de contexto se refiere no solo al entorno situacional, sino fundamentalmente a una configuración de interacciones sociales que involucran una textura discursiva, lingüística y multimodal, y significados compartidos.

El CSE debe ser entendido como una construcción multidimensional integrada por múltiples indicadores. Aunque se propusieron diferentes formas de analizar esta cuestión (Hill y Michael, 2001), aquí utilizamos algunos indicadores para distinguir los contextos socioeconómicos favorecido y desfavorecido (CSF y CSD, respectivamente): la ocupación de los padres (especialmente el jefe de familia), nivel educativo de los padres (particularmente la madre), ingreso familiar principal y condición habitacional (Arán Filippetti, 2012; Duncan y Magnuson, 2003; Ensminger y Fothergill, 2003). Consideramos estos indicadores de una manera global unitaria (Noble, Norman y Farah, 2005), sin descomponerlos en elementos diferenciados.

En general, el estudio de los procesos psicológicos en diferentes condiciones socioeconómicas se centró principalmente en la interacción madre-hijo (por ejemplo, Salsa y Peralta, 2001; Salsa y
Peralta, 2009) o procesos cognitivos individuales (entre otros, Arán Filipetti, 2012; Arán Filippetti y Richaud de Minzi, 2011; Lacunza, Contini y Castro, 2010; Lipina, Martelli, Vuelta, y Colombo, 2005; Salsa, 2012, 2013; Salsa y Gariboldi, 2017). Algunos de estos estudios describen las diferencias contextuales asociadas a funciones psicológicas específicas. Otros artículos analizan la implementación de programas de intervención para demostrar la posibilidad de recuperación cognitiva y la disminución de las diferencias entre los grupos sociales. La escuela de Ginebra desarrolló una serie de estudios, aunque no se continuaron después (por ejemplo, Doise y Mugny, 1984; Mugny y Doise, 1979). Esos experimentos, principalmente orientados a la recuperación cognitiva de niños de condiciones socioeconómicas desfavorables, demostraron el papel decisivo de la interacción social en el desarrollo: a partir de la implementación de sesiones de trabajo colaborativo se incrementaron los niveles cognitivos individuales y se redujo la brecha social inicialmente reportada.

\section{La perspectiva del análisis multidimensional de datos}

La versión francesa del análisis multidimensional de datos (AMD) surgió en los años setenta como una perspectiva con objetivos menos deterministas que las estadísticas tradicionales. La búsqueda de una estructura presente en los datos es el principio básico que guía la lógica de este análisis, en un contexto más inductivo que deductivo, que particularmente 
revaloriza el rol de cada observación individual. La forma descriptiva de proceder y la aproximación geométrica a los problemas otorgan un papel central a las representaciones gráficas de los datos (Moscoloni y Satriano, 2002).

El surgimiento del AMD se remonta al trabajo de Benzecri (1965), quien argumentó que el modelo debe seguir los datos y no la dirección opuesta tradicional. La propuesta de pasar a la multidimensionalidad provocó un importante cambio cualitativo: desde esta lógica, la realidad no solo se simplifica porque es compleja, sino que también se explora porque está oculta. Sin embargo, no se trata de presentar la información simplemente, sino también de analizar, descubrir y (en algunos casos) probar algunas hipótesis (Lebart, Morineau y Piron, 1995).

En el campo de las ciencias sociales, la problemática se convierte en una cuestión importante porque el estudio estadístico de las variables cualitativas (muy frecuentes en esta área) sería naturalmente más complejo que el estudio estadístico de las variables numéricas continuas. Las técnicas de AMD permiten un análisis más exhaustivo de los datos; lo que es posible por la velocidad de los cálculos y la percepción inmediata de la estructura de datos. También, permite la detección de relaciones entre variables que serían imperceptibles para los investigadores, cuando consideran simultáneamente una gran cantidad de variables cruzadas entre sí.

Específicamente en relación con el procesamiento de los datos, el uso de técnicas de AMD modifica profundamente las primeras fases del tratamiento: estas técnicas no son solo complementos que intervienen después de los métodos tradicionales, sino que cambian radicalmente el encadenamiento de etapas y definen una nueva metodología y conceptos (Moscoloni, 2005a). Una de las principales características del AMD es el retorno a la realidad en la interpretación de los datos: los resultados se expresan en unidades de medida consistentes con el objeto que permite un mayor reconocimiento de su complejidad (Moscoloni, 2004). Esto es particularmente importante en el campo de las ciencias sociales, donde el objeto presenta un mayor nivel de complejidad y no permite reducciones simples.

En este sentido, el AMD también permite explorar diferentes dimensiones del objeto. Por otro lado, disminuye el carácter confirmatorio de las estadísticas tradicionales probabilísticas. Aunque el enfoque de probabilidad no se rechaza, supone un objetivo diferente sobre la decisión final. Las pruebas estadísticas no se consideran como valores que rechazan o no las hipótesis nulas de los estudios, para convertirse en valores que ordenan las diferentes características de una clase. El investigador recupera su papel activo en la interpretación de los datos, especialmente a través de la visualización gráfica de la proyección espacial de los valores de variables y unidades de observación. Esas características distinguen al AMD de las estadísticas tradicionales (Moscoloni, 2005b).

El AMD propone dos grupos complementarios de métodos, utilizados en una secuencia de etapas que permite una 
reducción, sin perder de vista la complejidad de los datos: a) métodos factoriales que producen representaciones gráficas de las asociaciones estadísticas entre líneas y columnas, y b) métodos de clasificación que agrupan individuos con características similares. Según Moscoloni (2004), ambas técnicas se complementan a través de una serie de pasos, que se describen a continuación.

En primer lugar, el análisis factorial se realiza eligiendo tanto el conjunto de variables activas (que intervienen en la determinación de los ejes del análisis factorial), como el conjunto de variables ilustrativas (que se refieren a otras dimensiones de la variable o son opuestas a las activas). Luego, se debe proceder a la descripción gráfica de la población, donde la proximidad entre los individuos-observaciones corresponde a la similitud de sus respuestas respecto de las variables activas. La proyección de los puntos de los individuos-observaciones en el espacio de las variables debe ser correlacionada con el valor de sus coordenadas en ese espacio.

El segundo paso del procesamiento es la clasificación mixta en un subespacio factorial. Esta clasificación se realiza teniendo en cuenta los valores en cada punto de observación individual, respectivamente en cada eje factorial (a diferencia de las estadísticas clásicas que realizan la clasificación tomando los valores reales de cada individuo en cada una de las variables medidas en su escala original). De esta forma, se modera la posible distorsión causada por las diferentes escalas de medida y se trabaja con las oposiciones más importantes fundadas en los datos. En otras palabras, esta etapa hace referencia a la configuración de clases o clusters con características similares, que permiten la observación de las variables asociadas (Moscoloni, 2005).

En tercer lugar, el análisis describe estas clases de individuos-observaciones constituidas según sus características comunes. Entonces, las categorías características se dan a cada clase, facilitando la interpretación de cada grupo. En cuarto lugar, las clases están posicionadas en el plano factorial, lo que permite visualizar la localización en el espacio y la concentración de estas. Por último, una de las principales contribuciones del AMD es la importancia que se da a los individuos. En este sentido, permite estudiar casos con técnicas cualitativas, partiendo de los grupos construidos en la fase previa y, luego, elegir los individuos más característicos de cada clase para analizarlos profundamente como modelos, prototipos o parangones (Aluja y Morineau, 1999). Son los individuos más cercanos al centro de gravedad en cada grupo o clase. Esta proximidad se explica en términos de la distancia definida en el proceso de clasificación.

En resumen, los métodos factoriales son esenciales para alcanzar una visión global exhaustiva de una matriz de datos. La capacidad descriptiva de los ejes y la posibilidad de detectar tendencias los convierten en un importante procedimiento exploratorio de la estructura de datos. Simultáneamente, el análisis de clasificación es útil para interpretar los ejes factoriales (por su gran permeabilidad 
a la intuición), constituyendo grupos con características homogéneas dentro de ellos y heterogéneos entre sí, y localizando a los individuos más típicos en cada uno de estos conglomerados.

Para ilustrar la aplicación de la versión francesa del AMD, se utilizan los datos de una investigación previa (cita omitida para mantener el anonimato de los autores), cuyo objetivo fue analizar las características de la colaboración infantil entre pares, según la edad (cuatro, ocho y doce años) y el CSE, en una tarea de construcción con bloques y un dibujo libre. Aquí solo se incluyeron los datos referidos a la tarea de construcción.

La interacción fue analizada por un sistema de categorías sociocomportamentales exhaustivas y mutuamente excluyentes (unidades molares): disociación, dominio-sumisión, cooperación implícita, cooperación explícita, colaboración, conversación relacionada con la tarea sin ejecución, conversación ajena a la tarea sin ejecución. Además, estas categorías se complementaron con un segundo grupo de códigos verbales utilizados en otra publicación (cita omitida para mantener el anonimato de los autores), referidas específicamente al contenidos semántico de las verbalizaciones emitidas durante la interacción (unidades moleculares).

\section{Método}

\section{Participantes}

Participaron 82 sujetos (41 díadas) de tres escuelas de la ciudad de Rosario
(Argentina). Veintiséis eran de preescolar $(\mathrm{M}=4.7, \mathrm{SD}=0.3,12$ niñas y 14 niños), 28 niños eran de tercer año de primaria $(\mathrm{M}=8.7, \mathrm{SD}=0.3,12$ niñas y 16 niños) y 28 niños eran de séptimo año de primaria $(\mathrm{M}=12.6, \mathrm{SD}=0.4,10$ niñas y 18 niños). A su vez, 46 de estos casos pertenecían a CSF y los otros 36 , a CSD. Cada niño trabajó en una díada con un compañero del mismo curso escolar y género. Además, ambos se habían elegido mutuamente para realizar la tarea de colaboración (afinidad socioafectiva recíproca).

Los participantes de CSF provenían de dos escuelas privadas ubicadas en el centro de la ciudad. La mayoría de estos vivía en áreas urbanas con acceso a servicios públicos básicos (transporte, electricidad, alcantarillado, gas natural, etc.) y en casas o edificios de valor económico medio y alto. En general, sus padres eran profesionales, maestros, dueños o empleados de comercios y empleados del gobierno. Todos habían completado, al menos, la escuela secundaria, y en varios casos tenían educación universitaria. En cambio, los participantes de CSD asistían una escuela ubicada en una zona marginal de bajos recursos de la ciudad. El barrio estaba emplazado de manera irregular sobre terrenos fiscales, y no contaba con una clara demarcación del trazado vial (en Argentina, estas localizaciones urbanas son denominadas villas de emergencia o villas miseria). Las condiciones habitacionales eran desfavorables, puesto que la mayoría de las viviendas estaban construidas con chapa, madera y otros materiales endebles. Los padres de los 
niños eran principalmente trabajadores ocasionales de la construcción, jardinería y carpintería, vendedores ambulantes, recolectores de basura o desempleados. Solo en unos pocos casos contaban con un trabajo permanente. La mayoría de las madres eran amas de casa, aunque otras trabajaban en el servicio doméstico. La mayoría de los padres no había completado la escuela primaria.

\section{Procedimiento}

La tarea consistió en la construcción con bloques de una casa. La consigna fue explícitamente colaborativa, ya que se solicitaba a ambos niños que trabajen conjuntamente en la construcción de una casa, con base en los materiales proporcionados y tratando de tomar todas las decisiones respectivas de manera compartida. Los niños de ocho y doce años usaron 500 bloques tipo Lego de diferente forma, volumen y color. Los niños de doce años también usaron piezas y tejas opcionales para la construcción del techo. Los niños de cuatro años usaron los mismos materiales que los niños mayores, pero con adaptaciones evolutivas. Sus bloques eran más grandes, más flexibles y con un menor número de unidades (23 bloques). Éstos también tenían a disposición un modelo de casa, que se usó como referencia para la construcción. Todas estas adaptaciones evolutivas de los materiales se realizaron por una cuestión ecológica, con el fin de poder estudiar la interacción colaborativa a tan temprana edad y en las condiciones propias de cada momento del desarrollo.
La interacción fue grabada en video y transcrita en sus aspectos verbales y no verbales

\section{Codificación y análisis de datos}

La interacción fue analizada en dos aspectos. Primero, siguiendo a los autores anónimos (cita omitida para mantener el anonimato de los autores), estudiamos la modalidad social de ejecución de la tarea, en función de tres indicadores: a) cuántos sujetos estaban ejecutando acciones sobre los materiales en un momento dado; b) en qué medida estas acciones estuvieron orientadas hacia un producto final común y colectivo, y c) en el caso de que ambos sujetos estén trabajando hacia un producto común, cómo se regulaba la distribución social de las funciones, es decir, espontánea o verbalmente. La interacción se dividió en intervalos regulares de 10 segundos. Cada intervalo fue codificado por solo una categoría. Un segundo observador codificó el $10 \%$ de los datos (selección probabilística). El nivel de acuerdo entre codificadores fue aceptable $(K=0.98)$. Los desacuerdos se resolvieron mediante discusión. Las categorías se describen a continuación.

Disociación (DI). Cada niño dirige sus acciones hacia un fin individual, no integrado al desarrollado por su compañero (dos producciones separadas). Esta categoría también se aplica cuando un niño dirige las acciones hacia el logro de un producto único, mientras que el otro no trabaja en los materiales y es indiferente con respecto a las acciones del primero. 
Dominio-sumisión (DO). Un niño monopoliza la ejecución de la tarea y agrega coercitivamente al otro a esta acción. También puede ocurrir que uno de los niños ejecute acciones para lograr un producto único mientras que el otro no trabaja sobre los materiales, pero participa pasivamente en la acción (por ejemplo, observación silenciosa y atención sostenida de la actividad individual del compañero).

Cooperación implícita (CPI). Ambos niños orientan sus acciones hacia un fin colectivo común. Cada uno de ellos ejecuta acciones de manera individual, para luego unirlas y lograr una integración final colectiva. Esta distribución de funciones es espontánea, es decir, sin una explicación verbal. Un indicador común de la cooperación espontánea es el monitoreo visual recurrente de cada niño respecto a las acciones de su compañero, como un mecanismo de coordinación alternativo al acuerdo verbal.

Cooperación explícita (CPE). Esta categoría es similar a la anterior, pero la asunción de funciones individuales específicas (que luego se integran en una producción colectiva) se basa en un acuerdo verbal previo.

Colaboración (CO). Ambos niños trabajan simultáneamente en la resolución conjunta de un mismo aspecto de la tarea (sin división de funciones, como en la cooperación). Participan de modo simétrico en la resolución de un mismo aspecto de la tarea, proponiendo alternativamente ideas, soluciones, sugerencias y correcciones.
Conversación relacionada con la tarea sin ejecución (CRT). Ningún sujeto ejecuta acciones concretas sobre los materiales, sino que dialogan con el fin de discutir y analizar posibles acciones o distribución de funciones, en ambos casos pertinentes a la resolución de la tarea.

Conversación ajena a la tarea sin ejecución (CAT). Ningún niño ejecuta acciones concretas sobre los materiales y emiten verbalizaciones no pertinentes a la realización de la tarea.

En segundo lugar, aunque las categorías de modalidad social de ejecución consideraban de manera secundaria los aspectos conversacionales, no incluyeron un análisis específico sobre el contenido semántico de los mensajes. Por lo tanto, se propuso un segundo análisis orientado puntualmente al contenido lingüístico de las verbalizaciones individuales (cita omitida para mantener el anonimato de los autores) durante la interacción.

La unidad de análisis fue cada turno conversacional interrumpido por el compañero o porque finalizaba espontáneamente. Cada mensaje fue codificado por solo una categoría verbal. Un segundo observador codificó el $10 \%$ de los datos (selección probabilística). El nivel de acuerdo entre codificadores fue aceptable $(90 \% ; \mathrm{K}=$ 0,86). Los desacuerdos se resolvieron mediante discusión. Las categorías verbales se presentan a continuación.

Aporte cognitivo individual (ACI). Mensaje directamente relacionado con la resolución de un aspecto central de la tarea, sin consultar e intentar previamente un acuerdo con el compañero 
("Acá tenemos que meter la puerta, dejando un espacio").

Búsqueda de acuerdo (BA). Pregunta o comentario al compañero destinado a tomar una decisión consensuada relativa a la resolución de un aspecto de la tarea. También incluye las respuestas dadas por el compañero ante la búsqueda de consenso del primero y otros mensajes posteriores que guardan relación con el marco vincular mencionado ("¿De cuántos bloques querés hacer la altura de la pared?").

Solicitud de ayuda (RA). Mensaje dirigido al compañero con el fin de solicitar su ayuda, a partir de dificultades propias para resolver un aspecto de la tarea o para encontrar una estrategia de resolución (¿Cómo se engancha esta ventana?).

Directiva (D). Mensaje orientado al compañero para inducirlo a efectuar una acción orientada a la resolución de la tarea. Esta categoría incluye: a) indicarle una acción específica porque el primero solicitó una explicación; b) corregir una acción motriz y la estrategia cognitiva del compañero; c) indicarle la realización de una acción directamente relacionada con la resolución de la tarea. (“Agregá un ladrillo más, así no queda tan chico").

Planificación (PLAN). Mensaje asociado a la previsión anticipada de una acción y estrategias mediatas destinadas a ser aplicadas durante el curso posterior de la tarea [“Hacemos así, mirá. Hacemos todo un borde así... (toma ladrillos y forma un perímetro para la casa) y después acá vamos levantando paredes"].
Mensaje organizativo inespecifico (MOI). Mensaje relacionado con la actividad, pero no directamente vinculado con la resolución lógica de la tarea, sino con la organización general de la acción colectiva ("En esta caja hay más ladrillos pequeños").

Distribución de funciones individuales (DF). Mensaje cuya finalidad es proponer y acordar explícitamente una distribución de roles y funciones (marco regulatorio social) para la resolución colectiva de la tarea ("Vos seguí uniendo las paredes, que yo hago el otro piso y las ventanas, ¿dale?”).

Mensaje ajeno a la tarea (MAJ). Mensaje cuyo contenido no está relacionado con el objetivo de la tarea ("Hay sol de nuevo").

Con respecto a la modalidad social de ejecución, cada díada se codificó según la categoría más observada durante la tarea total. Cuando una de las categorías ocupaba $>50 \%$ de los intervalos regulares, la díada se codificó como tal (por ejemplo, la díada registró $80 \%$ de cooperación explícita, se codificó como CPE). Sin embargo, si no había ninguna categoría que ocupaba $>50 \%$ de los intervalos, la díada recibía un código mixto según la modalidad de interacción más frecuente [por ejemplo, si las categorías más frecuentes en una díada eran cooperación explícita $(30 \%)$ y colaboración (45\%), se codificaba como $\mathrm{CPE} / \mathrm{CO}$ ). Por otro lado, en lo que se refiere a las categorías de verbalización específicas, se dividió la frecuencia total de cada tipo de mensaje por el tiempo total de la tarea. De 
esta manera, cada categoría verbal se consideró como una variable continua, independientemente de las demás.

\section{Resultados}

La aplicación del AMD apuntó a considerar simultáneamente todas las categorías propuestas. Para ello se utilizó la versión Software SPAD® 5.6 (Système Portable pour l'Analyse de Données). La modalidad de interacción y el CSE se consideraron como variables nominales activas; la edad fue considerada como variable nominal ilustrativa; cada categoría de mensaje (independiente) se consideró como variable ilustrativa continua. El análisis factorial de correspondencia múltiple mostró los siguientes resultados.

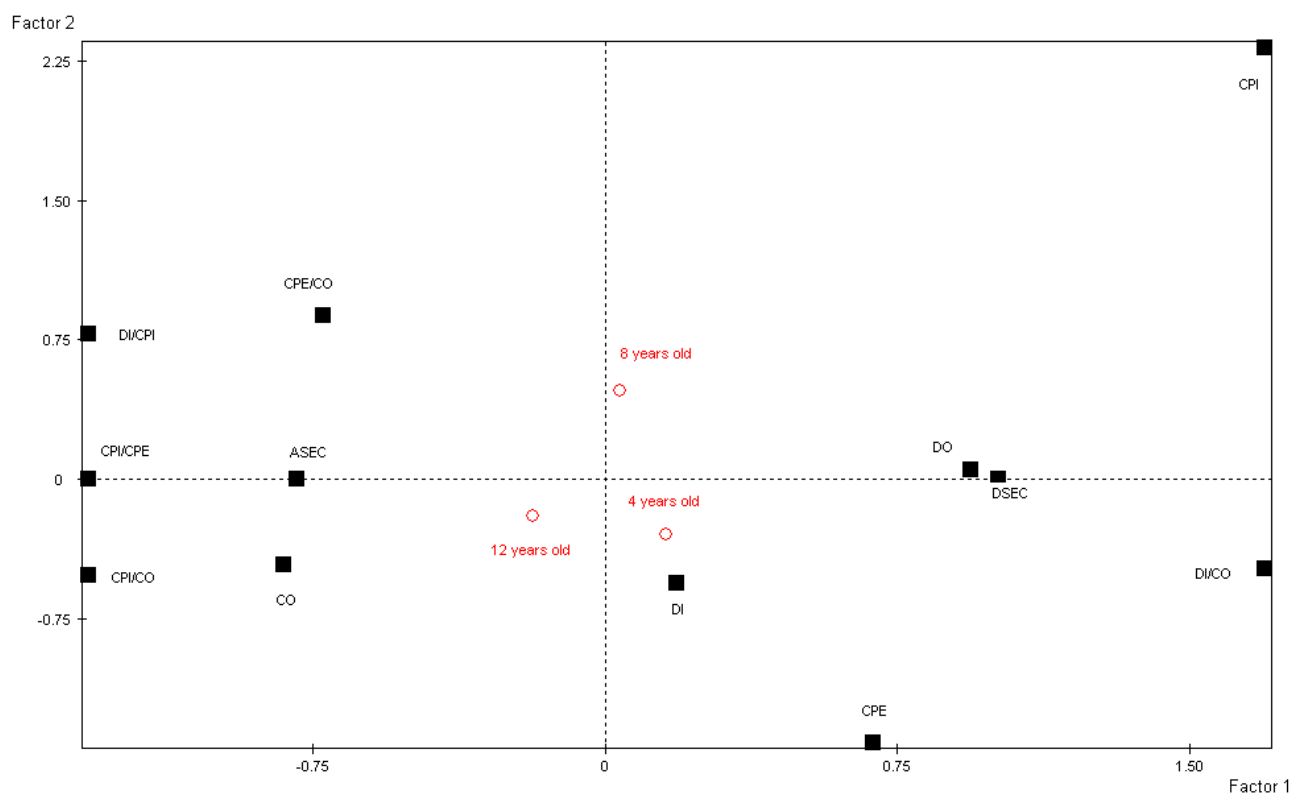

Nota: $\mathrm{ASEC}=\mathrm{CSF}$ (contexto socioeconómico favorecido) $/ \mathrm{DSEC}=\mathrm{CSD}$ (contexto socioeconómico desfavorecido).

Figura 1. Proyección de las variables nominales activas e ilustrativas en los ejes factoriales 
Tabla 1

Valores estadísticos de las variables activas nominales e ilustrativas continuas

\begin{tabular}{|c|c|c|c|}
\hline \multicolumn{4}{|c|}{ FACTOR 1} \\
\hline Variables activas nominales & Modalidad & Valor test & Carga \\
\hline Contexto socioeconómico & CSF & -5.65 & 23.00 \\
\hline Modalidad de interacción & $\mathrm{CPE} / \mathrm{CO}$ & -2.59 & 10.00 \\
\hline Modalidad de interacción & $\mathrm{CO}$ & -2.16 & 6.00 \\
\hline Variables ilustrativas continuas & & Coordenadas & Carga \\
\hline MOI & & -0.55 & 41.00 \\
\hline BA & & -0.42 & 41.00 \\
\hline \multicolumn{4}{|l|}{ ÁREA MEDIA } \\
\hline Variables activas nominales & Modalidad & Valor test & Carga \\
\hline Modalidad de interacción & $\mathrm{CPI}$ & 3.01 & 3.00 \\
\hline Contexto socioeconómico & CSD & 5.65 & 18.00 \\
\hline Variables ilustrativas continuas & & Coordenadas & Carga \\
\hline RA & & 0.01 & 41.00 \\
\hline \multicolumn{4}{|c|}{ FACTOR 2} \\
\hline Variables activas nominales & Modalidad & Valor test & Carga \\
\hline Modalidad de interacción & CPE & -3.73 & 6.00 \\
\hline Variables ilustrativas continuas & & Coordenadas & Carga \\
\hline PLAN & & -0.20 & 41.00 \\
\hline MAJ & & -0.07 & 41.00 \\
\hline \multicolumn{4}{|l|}{ ÁREA MEDIA } \\
\hline Variables activas nominales & Modalidad & Valor test & Carga \\
\hline Modalidad de interacción & $\mathrm{CPE} / \mathrm{CO}$ & 3.18 & 10.00 \\
\hline Modalidad de interacción & $\mathrm{CPI}$ & 4.12 & 3.00 \\
\hline Variables ilustrativas continuas & & Coordenadas & Carga \\
\hline $\mathrm{MOI}$ & & 0.00 & 41.00 \\
\hline D & & 0.01 & 41.00 \\
\hline
\end{tabular}

Como puede observarse en la figura 1 , en el factor 1 (o eje horizontal) prevalece la condición socioeconómica. Hacia la derecha, se concentra la modalidad CSD de la variable activa condición socioeconómica, mientras que a la izquierda se concentra la modalidad CSF. Se destaca una asociación entre CSD con la modalidad CPI; es decir, la división de tareas donde cada participante asume una función específica de manera espontánea, sin apelar a la explicitación verbal. Mientras, el CSF se vincula con CPE y $\mathrm{CO}$; o sea, a la forma explícita de división de tareas y asunción de funciones en la realización de la actividad y a la colaboración propiamente dicha, cuyo fin es la realización de un producto colectivo. 
Este primer factor visibiliza un resultado de importancia teórica relevante. La condición socioeconómica no se vincularía con la ausencia/presencia de cooperación o colaboración, sino que se destaca la existencia de las modalidades de mayor integración de coordinación social $(\mathrm{CO} / \mathrm{CPE} / \mathrm{CPI})$ y se opone solamente lo implícito/explícito. Es decir, este eje está determinado por la presencia (CPE) o ausencia (CPI) de una mediación semiótica de acuerdos en la distribución de tareas.

El factor 2 (o eje vertical) está determinado principalmente por las modalidades de mayor integración social $(\mathrm{CPI} / \mathrm{CPE} / \mathrm{CO})$ de la variable activa modalidad de interacción. Hacia arriba se observan las modalidades CPI, CPE y $\mathrm{CO}$ y, hacia abajo, solo CPE. Este eje tendería a reproducir la clásica distinción teórica entre cooperación y colaboración. Mientras que la agrupación de las modalidades CPI, CPE y CO hace referencia en su conjunto a las distintas instancias grupales para acceder a la verdadera colaboración (planificación mental de la tarea, la explicitación de funciones y la consecución de un objetivo común), el segundo extremo destaca la cooperación propiamente dicha; o sea, la realización de la actividad sostenida en un consenso simbólico previo.

La tabla que se incluye a continuación muestra los valores estadísticos de los factores antes descritos y las variables suplementarias continuas incluidas en el análisis.

\section{Tabla 2}

Descripción de las clases y las principales variables definitorias de los ejes factoriales

\begin{tabular}{|c|c|c|c|c|c|}
\hline Clase & $N(\%)$ & $\begin{array}{c}\text { Modalidad de la variable } \\
\text { con mayor carga }\end{array}$ & $\begin{array}{l}\text { Porcentaje de la catego- } \\
\text { ría en la clase }\end{array}$ & Valor test & Probabilidad \\
\hline \multirow[t]{2}{*}{1} & 56.10 & CSF & 91.30 & 5.08 & .000 \\
\hline & & $\mathrm{CPE} / \mathrm{CO}$ & 43.48 & 3.08 & .001 \\
\hline 2 & 7.32 & CPI & 100.00 & 3.74 & .000 \\
\hline \multirow[t]{2}{*}{3} & 36.59 & CSD & 86.67 & 3.97 & .000 \\
\hline & & CPE & 40.00 & 3.06 & .001 \\
\hline
\end{tabular}

Como puede observarse, los clústeres 1 y 3 responden a los datos arrojados por el análisis factorial. El clúster 1 se caracteriza por la condición socioeconómica favorecida $\mathrm{y}$, vinculadas a ella, las modalidades de integración social mediadas por el lenguaje. El clúster 3 vincula los sujetos de CSD y, en menor medida, la cooperación explícita. Mientras que el clúster 2, de menor tamaño y determinado por la CPI; 
es decir, la modalidad de cooperación implícita, está formado por tres casos vinculados entre sí por la categoría CPI. Gracias al análisis multidimensional de datos, se pudieron detectar estos tres casos o parangones, elaborar una descripción de ellos y vislumbrar sus particularidades. De esta manera, se observó que los tres casos eran sujetos de ocho años de CSD, cuya modalidad de interacción era CPI. Estos datos vuelven a corroborar lo planteado en los ejes factoriales anteriores.

\section{DISCUSIÓN}

En este artículo se analizó la relación entre colaboración infantil entre pares y CSE desde la perspectiva propuesta por el AMD, en su versión francesa. Podríamos decir que los resultados son coherentes con respecto a trabajos previos basados en una perspectiva sociocultural. Las principales diferencias contextuales en la colaboración entre pares podrían estar relacionadas con la modalidad, y no tanto con el grado, de coordinación social. Por ejemplo, los niños de CSF y CSD mostraron un alto nivel de coordinación social en la tarea de construcción, pero la principal diferencia fue que los primeros tienden a usar acuerdos verbales, mientras que los segundos principalmente llevan a cabo formas espontáneas (no verbales) de integración intersubjetiva.

Estas conclusiones son similares a trabajos anteriores (Correa-Chávez et al., 2016; Mejía-Arauz et al., 2012), aunque estos compararon sujetos de otras poblaciones, por ejemplo, niños urbanos e indígenas, mientras el presente trabajo se refirió en su totalidad a sujetos urbanos diferenciados por indicadores socioeconómicos. Más allá de esta diferencia, este trabajo y dichos antecedentes comparten un mismo marco conceptual sociocultural, que enfatiza las formas interactivas diarias asociadas al contexto más que al conjunto de aspectos externos que lo definen.

Al mismo tiempo, los resultados se refieren a la distinción clásica entre cooperación y colaboración que está, a su vez, vinculada a diferentes teorías conceptuales sobre la interacción sociocognitiva (Dillembourg, 1999, Barkley, Cross y Mayor, 2005). La colaboración alude a la constitución de un sujeto colectivo desde el inicio, mientras que la cooperación se refiere a una distribución de las funciones individuales, seguida por la integración de estas obras parciales individuales y el logro de una producción colectiva.

Por otro lado, tal como se ha visto, la versión francesa de AMD (Benzecri, 1965) proporciona una forma alternativa de procesar datos. Y supone una aproximación compleja al fenómeno, ya que se consideran simultáneamente diferentes aspectos o variables. Como dijimos, una de las principales características del AMD es el retorno a la realidad en la interpretación de datos, expresando los resultados en unidades de medida consistentes con el objeto y permitiendo un mayor reconocimiento de su complejidad (Moscoloni, 2004). Esto es particularmente importante en el campo de las ciencias sociales, donde el objeto presenta un mayor nivel de complejidad y no permite reducciones simples, al proporcionar una perspectiva exploratoria sobre los datos. 
Entre las principales limitaciones del trabajo, vale destacar el tamaño de la muestra, puesto que la aplicación del análisis multivariado es más apropiada con una mayor cantidad de participantes. Sin embargo, al mismo tiempo, consideramos que el AMD es una buena opción para realizar el procesamiento cuantitativo de datos, incluidas las muestras pequeñas, que son muy frecuentes en investigaciones clínicas o casuísticas en Psicología. El AMD facilita el reconocimiento de las tendencias generales de los datos (por ejemplo, mediante la interpretación de ejes factoriales o clústeres) y, luego, la detección de casos típicos y atípicos.

\section{RefERENCIAS}

Aluja T., y Morineau A. (1999). Aprender de los datos: el análisis de componentes principales. Una aproximación desde el data mining. Barcelona: EUB.

Arán Filippetti, V. (2012). Estrato socioeconómico y habilidades cognitivas en niños escolarizados: Variables predictoras y mediadoras. Psykhe, 21(1), 3-20.

Arán Filippetti, V., y Richaud de Minzi, C. (2011). Efectos de un programa de intervención para aumentar la flexibilidad y la planificación en un ámbito escolar de alto riesgo por pobreza. Universitas Psychologica, 10(2), 341-354.

Barkley, E. F., Cross, K. P., y Major, C. H. (2005). Collaborative learning techniques: A handbook for college faculty. San Francisco, USA: Jossey-Bass.

Castellaro, M., y Roselli, N. (2015a). Peer collaboration in childhood according to age, socioeconomic context and task. European Journal of Psychology of Education, 30, 63-80.

Castellaro, M., y Roselli, N. (2015b). La comunicación verbal colaborativa en tres grupos etarios y dos contextos socioeconómicos. Revista Latinoamericana de Ciencias Sociales, Niñez y Juventud, 13(2), 879-891.

Correa-Chávez, M., y Rogoff, B. (2009). Children's attention to interactions directed to others: Guatemalan Mayan and European American patterns. Developmental Psychology, 45(3), 630-641.

Correa-Chávez, M., Mangione, H., y Mejía Arauz, R. (2016). Collaboration patterns among Mexican children in an Indigenous town and Mexican City. Journal of Applied Developmental Psychology, 44, 105-113.

Correa-Chávez, M., Rogoff, B., y Mejía Arauz, R. (2005). Cultural patterns in attending to two events at once. Child Development, 76(3), 664-678.

Dillenbourg, P. (1999). What do you mean by “collaborative learning"? In P. Dillenbourg (Ed.), Collaborative-learning: Cognitive and computacional approaches (pp. 1-19). Armsterdam, Netherlands: Pergamon Press. 
Doise, W., y Mugny, G. (1984). The social development of the intellect. Oxford, England: Pergamon.

Duncan, G. J., y Magnuson, K. A. (2003). Off with Hollingshead: Socioeconomic resources, parenting, and child development. En M. H. Bornstein y R. H. Bradley (Eds.), Socioeconomic status, parenting, and child development (pp. 83-106). Mahwah, New Jersey: Lawrence Erlbaum.

Ensminger, M. E., y Fothergill, K. E. (2003). A decade of measuring SES: what it tells us and where to go from here. En M. H. Bornstein y R. H. Bradley (Eds.), Socioeconomic status, parenting, and child development (pp. 13-27). Mahwah, New Jersey: Lawrence Erlbaum.

Hill, C., y Michael, R. (2001). A new approach to measuring poverty. Journal of Human Resources, 36(4), 148-161.

Lacunza, A., Contini, N., y Castro, A. (2010). Las habilidades cognitivas en niños preescolares. Un estudio comparativo en un contexto de pobreza. Acta Colombiana de Psicología, 13(1), 25-34.

Lebart, L., Morineau, A., y Piron, M. (1995). Statistique Exploratoire Multidimensionelle. París: Dunod.

Lipina, S., Martelli, M., Vuelta, B., y Colombo, J. (2005). Performance on the A-not-B task of Argentinian infants from unsatisfied and satisfied basic needs homes. Interamerican Journal of Psychology, 39(1), 49-60.

Mejía-Arauz, R. (1999). El diálogo en la interacción colaboradora. Sinéctica, 14, 1-9.

Mejía-Arauz, R. (2015). Contrastes en el desarrollo sociocognitivo de niños en contextos urbanos y rurales o indígenas de México. En R. Mejía-Arauz (Coord.), Desarrollo Psicocultural de Niños Mexicanos (pp. 13-43). Guadalajara, México: ITESO.

Mejía-Arauz, R., Roberts, A., y Rogoff, B. (2012). Cultural variation in balance of nonverbal conversation and talk. International Perspectives in Psychology: Research, Practice, Consultation, 1(4), 207-220.

Mejía-Arauz, R., Rogoff, B., Dexter, A., y Najafi, B. (2007). Cultural variation in children's social organization. Child Development, 78(3), 1001-1014.

Moscoloni, N. (2004). El análisis multidimensional de datos como herramienta metodológica en ciencias sociales. VI Jornadas de Sociología. Facultad de Ciencias Sociales, Universidad de Buenos Aires, Buenos Aires.

Moscoloni, N. (2005a). Complementación metodológica para el análisis de datos cuantitativos y cualitativos en evaluación educativa. Revista electrónica de metodología aplicada, 10(2), 1-10. 
Moscoloni, N. (2005b). Las nubes de datos. Métodos para analizar la complejidad. Rosario: UNR editora.

Moscoloni, N., y Satriano, C. (2002). Construcción y tratamiento del "dato" en un estudio sobre desnutrición infantil. Cinta moebio. Revista de Epistemología de Ciencias Sociales, 13, 93-114.

Mugny, G., y Doise, W. (1979). Factores sociológicos y psicosociológicos en el desarrollo cognitivo: Una nueva ilustración experimental. Anuario de Psicología, 21(2), 3-26.

Noble, K. G., Norman, M. F., y Farah, M. J. (2005). Neurocognitive correlates of socioeconomic status in kindergarten children. Developmental Science, 8, 74-87.

Psaltis, C., Duveen, G., y Perret Clermont, A. (2009). The Social and the Psychological: Structure and context in intellectual development. Human Development, 52(5), 291-312.

Rogoff, B. (1990). Apprenticeship in thinking. Cognitive development in social context. New York, USA: Oxford University Press.

Rosemberg, C., Menti, A., Stein, A., Alam, F., y Migdalek, M. (2016). Vocabulario, narración y argumentación en los primeros años de la infancia y la niñez. Una revisión de investigaciones. Revista Costarricense de Psicología, 35(2), 101-120.

Salsa, A. (2012). Factores que influyen en la comprensión temprana de imágenes: Similitud perceptual y nivel socioeconómico. Infancia y Aprendizaje, Journal for the Study of Education and Development, 35(3), 313-326.

Salsa, A. (2013). Comprensión y producción de representaciones gráficas: Cambios evolutivos y diferencias por nivel socioeconómico. Cultura y Educación, 25(1), 95-108.

Salsa, A., y Peralta, O. (2001). Interacción materno-infantil con libros de imágenes en dos niveles socioeconómicos. Infancia y Aprendizaje, Journal for the Study of Education and Development, 24(3), 325-340.

Salsa, A., y Peralta, O. (2009). La lectura de material ilustrado: Resultados de una intervención con madres y niños pequeños de nivel socioeconómico bajo. Infancia y Aprendizaje, Journal for the Study of Education and Development, 32(1), 3-16.

Salsa, A., y Gariboldi, B. (2017). Experiencia con símbolos y comprensión de dibujos en niños pequeños de distintos contextos socioeconómicos. Avances en Psicología Latinoamericana, 36(1), 29-43 